\title{
Scanning for changes, recent PHENIX results from the beam energy scan at RHIC
}

\author{
Achim Franz* \\ for the PHENIX Collaboration \\ Brookhaven National Laboratory, Upton, NY 11973-5000, USA \\ E-mail: afranz@bnl.gov
}

\begin{abstract}
Recent QCD calculations [1] indicate that there should be areas in the phase diagram of nuclear matter accessible by high energy heavy-ion collisions, like at RHIC or LHC, where matter follows a smooth transitions from a Quark-Gluon-Plasma (QGP) phase back to hadronic matter, compared to lower beam energies, SPS and below, when it follows a first order phase transition. This suggests that there might be a critical point (CP) between these transition modes [2] somewhere between the highest SPS and RHIC energies. To find this CP RHIC has begun a beam energy scan (BES) [3] program from the highest heavy ion energies of $\sqrt{s_{N N}}=200 \mathrm{GeV}$ to the lowest so far of $\sqrt{s_{N N}}=7.7 \mathrm{GeV}$ with a possibility to go to $\sqrt{s_{N N}}=5 \mathrm{GeV}$, well below the standard injection energy of RHIC.
\end{abstract}

This paper summarizes some of the recent PHENIX results on global variables, the nuclear modification factor, and flow from this energy scan program.

Sixth International Conference on Quarks and Nuclear Physics,

April 16-20, 2012

Ecole Polytechnique, Palaiseau, Paris

\footnotetext{
* Speaker.
} 


\section{Introduction}

The Relativistic Heavy Ion Collider (RHIC) [4] at Brookhaven National Laboratory (BNL) is currently the only operating collider in the US. In 2012 it not only provided the highest energy collisions for polarized protons at $\sqrt{s_{N N}}=510 \mathrm{GeV}$, but also the first collisions of Uranium on Uranium at $\sqrt{s_{N N}}=192.8 \mathrm{GeV}$ and Copper on Gold at $\sqrt{s_{N N}}=200 \mathrm{GeV}$ thanks to its new Electron Beam Ion Source (EBIS) [5].

The last 11 years have brought exciting results including that the generated hot dense matter is not a weakly interacting gas but behaves more like a very opaque, strongly interacting liquid with a viscosity close to the quantum dynamical limit [6].

To study the different phases the RHIC BES program set out to access different areas in the matter diagram and maybe get close to the predicted CP. As the existence and location is still in question, experiments are comparing the results from different beam energies to scan for changes.

\section{Results}

\subsection{Global Variables}

Every time a new collider is brought into operation or a new energy regime is reached the first results involve global variables like charged particle multiplicity, momentum spectra and transverse energy flow. All these depend on the available beam energy and will of course increase as $\sqrt{s_{N N}}$ increases.

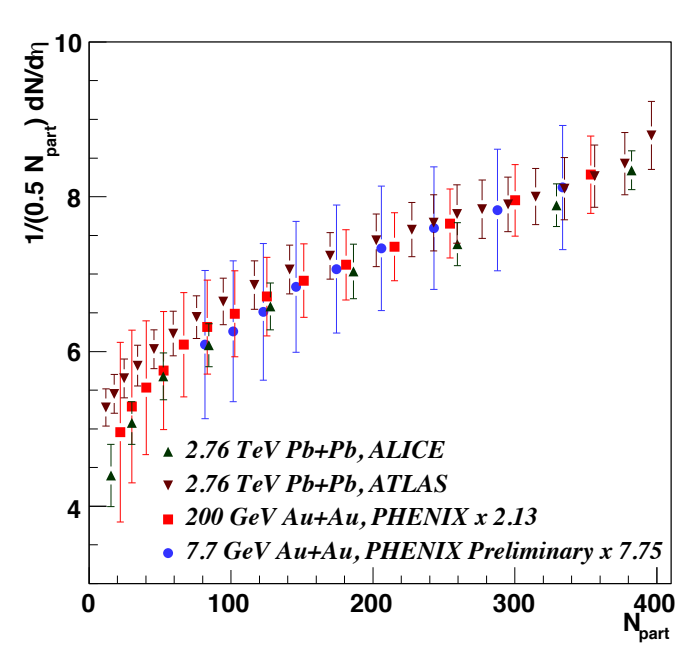

(a) Charged particles multiplicity per unit rapidity as a function of $N_{\text {part }}$ for RHIC and LHC beam energies

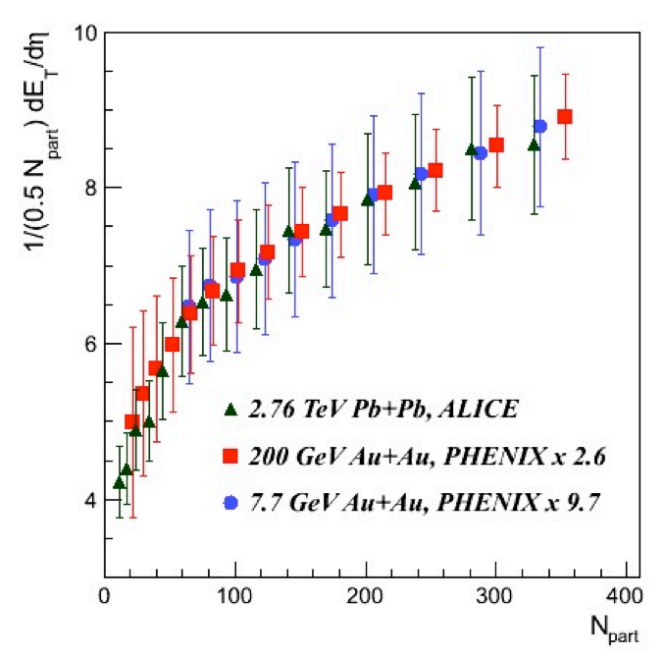

(b) Transverse energy sum per charge particles per unit rapidity for RHIC and LHC beam energies

Figure 1: Charged particle multiplicity and transverse energy comparisons

More interesting is how these values depend on the centrality of the collision which is determined by Glauber Monte-Carlo [7] comparisons to experimental data. Figures 1a and 1b [8] show the charged particle multiplicity and transverse energy at mid-rapidity from RHIC and LHC beam 
energies. When properly scaled, as indicated in the figures, the variation with centrality or number of participants in this case is very close. This clearly shows that the geometry of the collision is dominant from $\sqrt{s_{N N}}=0.0077 \mathrm{TeV}$ to $2.76 \mathrm{TeV}$ and the larger beam energy just increases the overall scale.

\subsection{Flow}

One of surprises of early RHIC running was the strong anisotropic flow observed in noncentral heavy-ion collisions which describes the azimuthal momentum space anisotropy of particle emission relative to the event plane $\Psi_{R P}$ expressed by the Fourier harmonics $v_{n}=<\cos \left[n\left(\phi_{i}-\Psi_{R P}\right)\right]>[9]$.

It was found that $v_{2}$ for baryons and mesons scales perfectly as a function of $K E_{T}=m_{T}-m_{0}$ when both variables are scaled by the number of constituent quarks indicating that the flow was parton based not hadronic. If at lower beam energies this partonic flow does not hold it would indicate a different phase or the non-creation of a QGP.
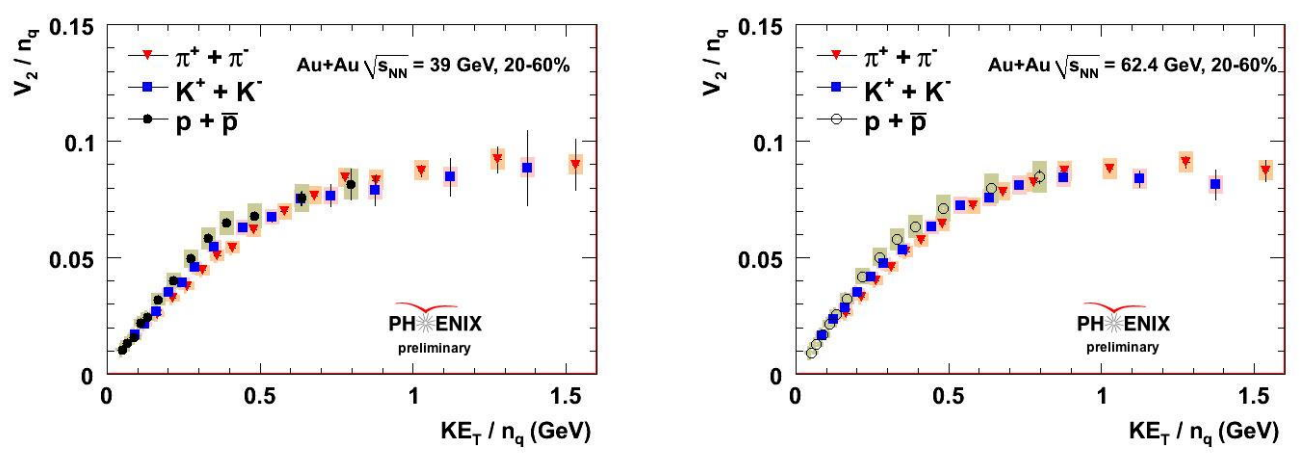

(a) Flow coefficients $v_{2} / n_{q}$ for $39 \mathrm{GeV} \mathrm{Au}+\mathrm{Au}$ (b) Flow coefficients $v_{2} / n_{q}$ for $62 \mathrm{GeV} \mathrm{Au+Au}$ collisions versus $K E_{T} / n_{q}$ where $n_{q}$ is the number collisions versus $K E_{T} / n_{q}$ where $n_{q}$ is the number of constituent quarks. of constituent quarks.

Figure 2: Flow coefficients $v_{2} / n_{q}$ versus $K E_{T} / n_{q}$

Figures. $2 \mathrm{a}$ and $2 \mathrm{~b}[10]$ show $v_{2}$ for $\mathrm{a} \sqrt{s_{N N}}$ of 39 and $62 \mathrm{GeV}$ for protons, kaons and pions scaled by the number of constituent quarks. These results indicate that down to $39 \mathrm{GeV}$ the quark number scaling still holds for the soft interaction region of $p_{T} \lesssim 3 \mathrm{GeV} / \mathrm{c}$ and we are still seeing a partonic flow, with a possible small deviation developing for protons at lower $p_{T}$ where the scaling might not be applicable.

Integrated over the available $p_{T}$ range, with the averages indicated in Fig. 3 (right), $v_{2}$ is constant over the $\sqrt{s_{N N}}$ range from 39 to $200 \mathrm{GeV}$ with only a moderate increase out to LHC energies [10]. More interesting is the drop off below $\sqrt{s_{N N}} \approx 30 \mathrm{GeV}$, see Fig. 3 (left). Upcoming RHIC results from $\sqrt{s_{N N}}$ of 19.6 and $27 \mathrm{GeV}$ should clarify where this drop begins. More on flow results from PHENIX can be found in the proceedings by E. Richardson in this volume.

\subsection{Nuclear modification factor}

Another important result from RHIC is the nuclear modification factor [11] where in central 

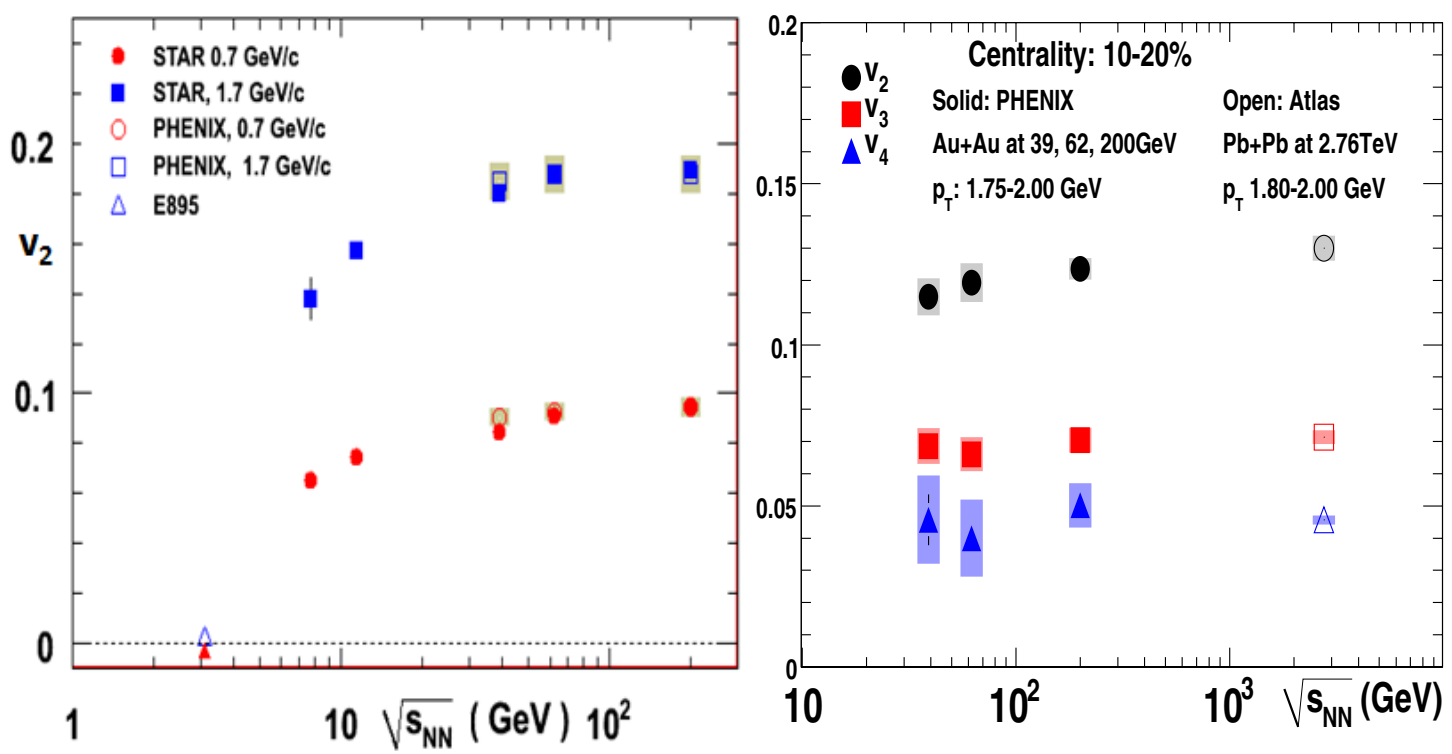

Figure 3: Integrated $v_{2}$ as a function of $\sqrt{s_{N N}}$, the average $p_{T}$ of the data is indicated in the figure.

nucleus-nucleus collisions high momentum particles are suppressed compared to participant scaled nucleon-nucleon collisions. PHENIX has measured this ratio for a variety of particles and beam energies [12]

Figure 4 [12] shows the $\pi^{0} R_{A A}$ from central $\mathrm{Cu}+\mathrm{Cu}$ collisions for three energies. The suppression levels are still large for the two higher beam energies, but at $\sqrt{s_{N N}}=22 \mathrm{GeV}$ for the lighter $\mathrm{Cu}+\mathrm{Cu}$ system, $R_{A A}$ shows an enhancement. A dataset of $\mathrm{Au}+\mathrm{Au}$ collisions at $\sqrt{s_{N N}}$ of 19.6 and $27 \mathrm{GeV}$ are being analyzed to see if they show a similar behavior.

\section{Summary}

PHENIX has taken data at several beam energies as part of the RHIC BES. So far in all analyzes no smoking gun for a critical point has been found. But many of the measurements especially at lower beam energies are limited in statistics which a future electron cooling for RHIC could solve. Some measurements show small deviations from higher to lower energies like the proton $v_{2}$ but require a more detailed analysis. 


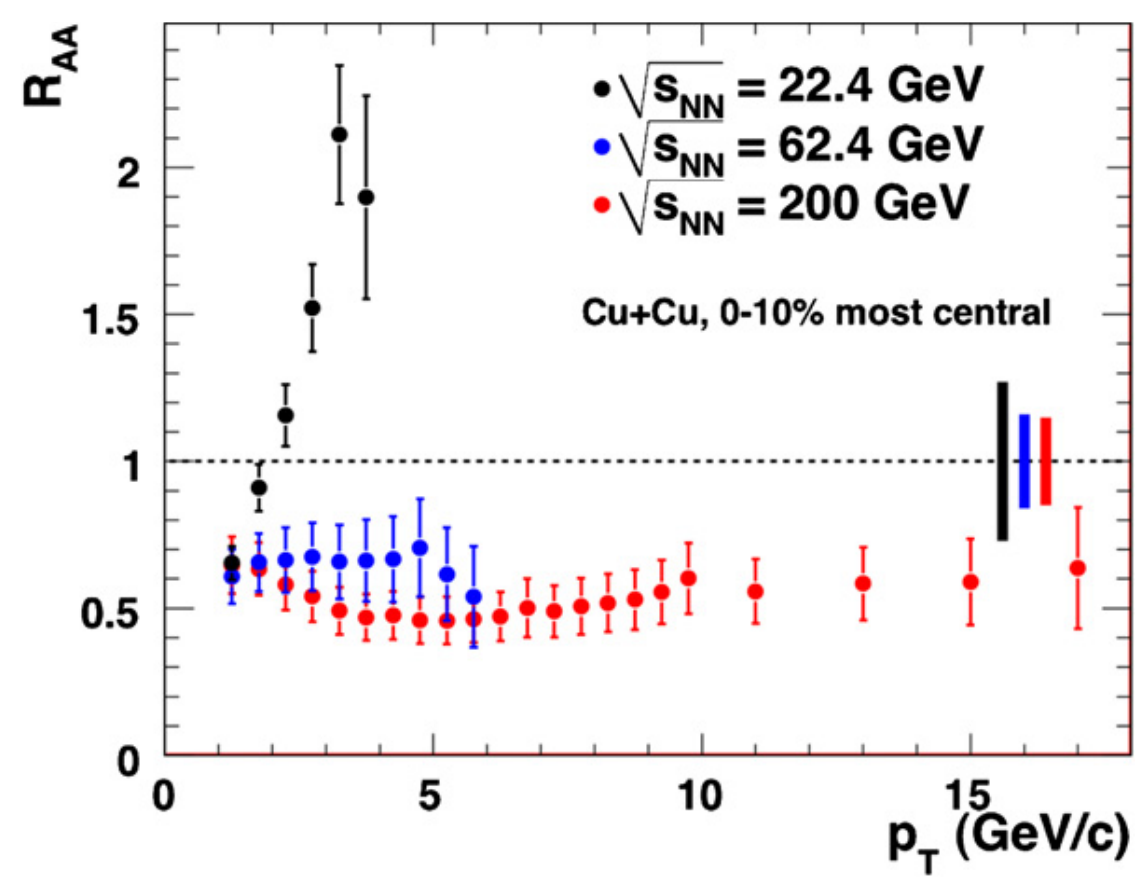

Figure 4: Integrated $R_{A A}$ for $\pi_{0}$ for central $\mathrm{Cu}+\mathrm{Cu}$ collisions as a function of $p_{T}$ for three beam energies

\section{References}

[1] U.Heller, PoS (LAT2006) 011 (2006) C.Schmidt, POS (LAT2006) 021 (2006) M.Alford, POS (LAT2006) 001 (2006)

[2] M. Stephanov, PoS (LAT2006) 024 (2006)

[3] T. Satogata, et al., POS (CPOD 0 7) 051

[4] M. Harrison, T. Ludlam and S. Ozaki, NIM A 499 Issues 2-3 (March 2003)

[5] A. Pikin et al., JINST 5 C09003 (2010)

[6] W.A. Zajc, Nucl.Phys. A 805, 283-294 (2008)

[7] M.L. Miller et al., Annu. Rev. Nucl. Part. Sci 57 (2007) 205-243

[8] J.T. Mitchell et al. PHENIX Collaboration, AIP Conf. Proc. 1422, 104 (2012)

[9] A.M. Poskanzer and S.A. Voloshin, Phys. Rev. C 58 (1998) 1671

[10] X.Gong et al., PHENIX Collaboration, J. Phys. G: Nucl. Part. Phys. 38(2011) 124146

[11] S.S. Adler et al. PHENIX Collaboration, Phys. Rev. Lett. 91, 072301 (2003)

[12] A. Adare et al. PHENIX Collaboration, arXiv:1204.1526v1 [nucl-ex] M.L. Purschke et al. PHENIX Collaboration, J. Phys. G: Nucl. Part. Phys. 38 (2011) 124016 